\title{
Calculated neutron energy dependence of the dose-response of large recombination chamber
}

\author{
Katarzyna Tymińska (1), \\ Michal A. Gryziński \\ Maciej Maciak
}

\begin{abstract}
A model of REM-2-type chamber was modeled with MCNPX code to study the dose-response to monoenergetic neutrons in wide energy range from thermal to $20 \mathrm{MeV}$ for various compositions of gas in the chamber. The energy dependence of the total dose absorbed in the filling gas was compared with the energy dependence of ambient absorbed dose $D^{*}(10)$ and with experimental data. The results of the studies will be useful for designing new, improved generation of recombination chambers.
\end{abstract}

Keywords: ambient absorbed dose $\bullet$ Monte Carlo $\bullet$ recombination chamber

K. Tymińska ${ }^{\boxplus}$, M. A. Gryziński

National Centre for Nuclear Research

7 Andrzeja Sołtana St., 05-400 Otwock-Świerk, Poland

E-mail: katarzyna.tyminska@ncbj.gov.pl

\section{Maciak}

Institute of Metrology and Biomedical Engineering

Warsaw University of Technology

8 Św. A. Boboli St., 02-525 Warsaw, Poland

Received: 13 April 2015

Accepted: 16 September 2019

\section{Introduction}

Recombination chambers are detectors devoted mainly for determination of dose equivalent in mixed radiation fields. The operation of the chamber is based on the fact that the experimentally determined dependence of ion collection efficiency on linear energy transfer (LET) can be correlated with the dependence of radiation quality factor on LET, Q(L). It means that, in principle, the chambers could be used as detectors for every kind of radiation, especially in the case of mixed radiation fields with complex composition and wide or unknown energy spectrum.

Among several types of recombination chambers, designed since their invention in the middle 1960s, the large, tissue-equivalent chamber of REM-2 [1] is the most suitable for determination of $\mathrm{H}^{*}(10)$.

The most popular method for determination of $H^{*}(10)$ with recombination chamber is based on determination of saturation current $\left(i_{\text {sat }}\right)$ and recombination index of radiation quality RIQ, denoted as Q4 [2]. The RIQ is derived [2-4] from the measurements of ion collection efficiency at a specially chosen polarizing voltage, so it expresses the amount of ion recombination, which depends on local ion density.

If the size of tissue-equivalent chamber can simulate the ICRU sphere, $\mathrm{H}^{*}(10)$ is well approximated by the product of the absorbed dose (proportional to the saturation current) and Q4.

$$
\dot{\mathrm{H}} *(10)=\frac{1}{R_{\text {cal }}} i_{\text {sat }} \mathrm{Q} 4
$$

where $R_{\text {cal }}$ is the response of the chamber, measured 
during calibration in a reference radiation field and expressed in terms of ambient dose equivalent rate.

$$
R_{\text {cal }}=\frac{\left(i_{\text {sat }} \mathrm{Q} 4\right)_{\text {cal }}}{\mathrm{H}^{*}(10)_{\text {cal }}}
$$

Like in any other detector, the response of REM-2 chamber in neutron radiation fields also depends on neutron energy and, to some extent, on composition of the radiation field [4]. It was shown in several studies [4-6] that the energy dependence of the chamber response is practically not crucial for radiation protection measurements. The values of $\mathrm{H}^{*}(10)$ determined in neutron radiation fields of broad energy spectra and in complex mixed radiation fields were usually in agreement with the reference values, within $10 \div 20 \%$.

Up to now, the energy dependence of the chamber response has never been calculated; however, such calculations could be very useful for validation of recombination methods and for improvement of existing recombination chambers. Finally, Monte Carlo calculations are the basis for constructing new generation of recombination chambers. Two processes should be separated in such calculations - the energy dependence of the dose absorbed in the gas in the chamber cavity and the ion recombination, which depends on the secondary particles spectrum in the gas. The dose-response is of primary interest for optimization of the chamber design, whereas the value of RIQ depends mostly on the mass of tissue-equivalent material in the chamber.

This paper presents the results of Monte Carlo calculations of the dose-response in the energy range from $0.01 \mathrm{eV}$ to $19 \mathrm{MeV}$.

The results were compared with experimental values, derived from previous measurements of $H^{*}(10)$ in reference with radiation fields at Physikalisch Technische Bundesanstalt (PTB) in Braunschweig [7].

\section{Numerical model of the REM-2 chamber}

Due to the lack of dedicated numerical tool for recombination chamber modeling, a procedure based on MCNPX code is proposed for modeling the chamber and calculation of the dose-response.

The detector is a cylindrical parallel-plate ionization chamber with 25 electrodes, made of quasi tissue-equivalent material containing $11 \%$ of hydrogen, $85.5 \%$ of carbon, and $3.5 \%$ of nitrogen, by weight. The electrodes have the same thickness of $3 \mathrm{~mm}$ and diameter of $12.2 \mathrm{~cm}$ but differ slightly in the shape, due to openings for rods connecting them alternately and thus forming two sets: current and voltage electrodes. The distance between the electrodes is $7 \mathrm{~mm}$. The cylinder is $42 \mathrm{~cm}$ in length and $16.5 \mathrm{~cm}$ in diameter. The mass of tissue-equivalent material in electrodes is $6.5 \mathrm{~kg}$ and the effective wall thickness is of about $2 \mathrm{~g} / \mathrm{cm}^{2}$. The chamber is enclosed in $1.2 \mathrm{~mm}$ thick duralumin case and covered with heat-insulating layer made of foamed polystyrene.

The model of the REM-2 chamber created using MCNPX code is shown in Fig. 1.

The space inside the chamber, filled with the gas, is a $26 \mathrm{~cm}$ high cylinder with diameter of $12.5 \mathrm{~cm}$,

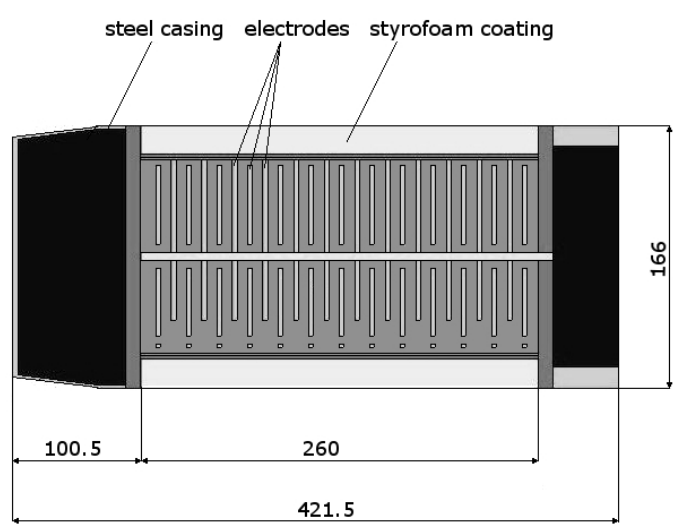

Fig. 1. The model of the REM-2 chamber created using MCNPX code.

forming gas volume of $2400 \mathrm{~cm}^{3}$. All the gas volume has been considered in the numerical model as active volume, while in real chamber the charge is collected only from the space formed by electrical field between the electrodes. Such approach considerably simplified the model. Taking into account that only some parts of secondary charged particles are generated in the gas, and that the difference between the two gas volumes mentioned above constitutes about $12 \%$ of the total gas volume, it can be expected that the influence of the simplification on the energy dependence of the chamber response is moderate.

The chamber is usually filled with a mixture of methane and nitrogen (5\%) up to the pressure of about $1 \mathrm{MPa}$ but the use of other gases and change of gas pressure is possible.

\section{Methods}

The chamber was modeled within the MCNPX code and used for flux calculations (MCNPX tally F2) of neutrons emitted from monoenergetic source in wide range of energy, up to $20 \mathrm{MeV}$ and then for calculations of total dose absorbed in gas (MCNPX tally $+\mathrm{F} 6$, scoring the energy deposited from all particles that are transported in the problem, over all energy ranges, in selected tally cells).

Neutrons were emitted from a cylindrical surface, placed concentrically around the chamber, providing efficient and symmetric source for the calculations. The number of particles generated was sufficient to obtain the relative uncertainties of the calculated dose below $1 \%$. Neutrons, photons, electrons, protons, and alphas were taken into account.

The gas filling the chamber was altered in subsequent simulations. There was methane with addition of nitrogen - from 5\% to $50 \%$ and ICRU methane-based tissue-equivalent gas [8].

The chamber filled with methane with $5 \%$ addition of nitrogen is a regular recombination chamber, which is able to measure $H^{*}(10)$ directly, even in an unknown radiation field. The calculations of the chamber response for other gases were performed at constant gas pressure of $1 \mathrm{MPa}$. A comparison with ICRU tissue-equivalent gas and comparison of gas mixtures with different hydrogen content were 
Table 1. Gas densities used for calculations

\begin{tabular}{ll}
\hline \multicolumn{1}{c}{ Composition of the gas } & $\begin{array}{l}\text { Density } \\
{\left[\mathrm{g} \cdot \mathrm{cm}^{-3}\right]}\end{array}$ \\
\hline Methane + nitrogen (5\% by weight) & 0.00744 \\
Methane + nitrogen (10\% by weight) & 0.00771 \\
Methane + nitrogen (20\% by weight) & 0.00825 \\
Methane + nitrogen (30\% by weight) & 0.00878 \\
Methane + nitrogen (50\% by weight) & 0.00986 \\
Tissue equivalent & 0.01290 \\
\hline
\end{tabular}

of main interest. Densities of the gases, used for calculations, are given in Table 1.

\section{Results}

The values of total dose absorbed in the chamber gas obtained in MCNPX calculations for monoenergetic neutrons are presented in Figs. 2 and 3, as the ratio of the calculated dose $(\mathrm{Dg})$ to the ambient dose, $D^{*}(10)$, which were derived from the literature data on ambient dose equivalent $\mathrm{H}^{*}(10)$ and radiation quality factor in ICRU sphere $Q^{*}(10)$, as $D^{*}(10)$ $=\mathrm{H}^{*}(10) / \mathrm{Q}^{*}(10)[9,10]$. Figure 2 presents the results for energy range from $0.075 \mathrm{MeV}$ to $19 \mathrm{MeV}$,

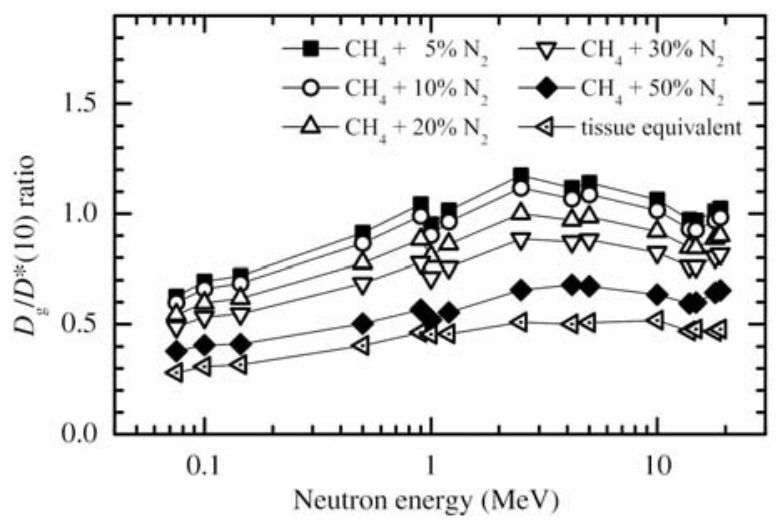

Fig. 2. The ratio of the calculated dose absorbed in gas $(\mathrm{Dg})$ to $\mathrm{D}^{*}(10)$ as a function of neutron energy for various gas compositions. The energy range corresponds to the energy range where the experimental values are available [7].

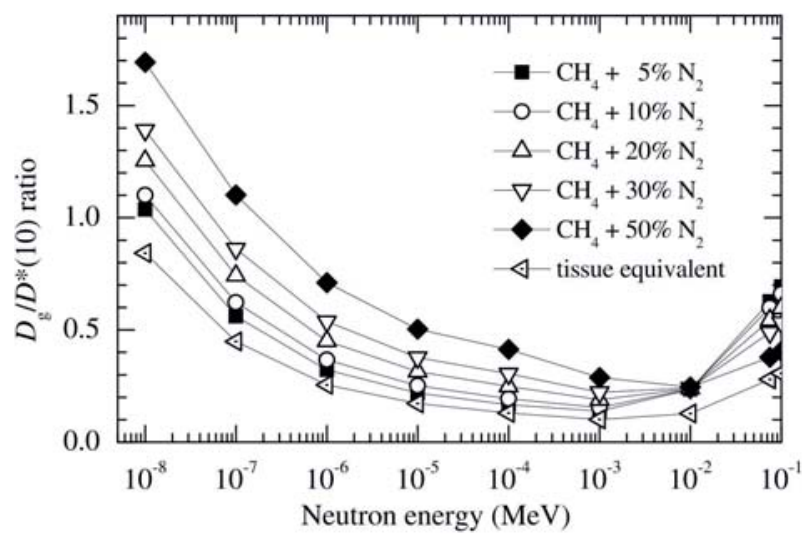

Fig. 3. The ratio of the calculated dose absorbed in gas $(\mathrm{Dg})$ to $\mathrm{D}^{*}(10)$ as a function of neutron energy for various gas compositions. The calculation results are presented for low energy range where the experimental results are not available. where also the experimental values are available [7]. Figure 3 shows the results for lower energies.

\section{Comparison with experimental data}

The influence of the filling gas composition on the chamber response has been earlier investigated using the commercial REM-2 chamber and the chamber of the same type but filled with tissue-equivalent gas mixture [4]. The measurements were performed for two isotopic neutron sources - ${ }^{241} \mathrm{Am}-\mathrm{Be}$ and ${ }^{239} \mathrm{Pu}-\mathrm{Be}$. For both sources, the response of the chamber filled with TE gas was lower by factor of $1.47 \pm 0.15$. This value is in at least qualitative agreement with the results of present calculations.

Another set of experimental data is the energy dependence of the chamber response to $H^{*}(10)$ of monoenergetic neutrons, which has been determined in reference radiation fields at Physikalisch Technische Bundesanstalt (PTB) in Braunschweig [7]. The measurements were performed for neutron energies ranging from $0.075 \mathrm{MeV}$ up to $19 \mathrm{MeV}$ using the chamber filled with the mixture of methane and $5 \%$ of nitrogen. The energy dependence of the dose-response, needed for this work, has been derived from these data by multiplying them by the ratio of Qref to Q4, where Q4 is a measured value of recombination index of radiation quality and Qref is the reference value of radiation quality factor, used for calculation of the reference $H^{*}(10)$ value. Unfortunately, the values of Qref have not been published, so they were estimated from the literature data on $Q^{*}(10)$ for neutrons, taking into account also the photon contribution to the absorbed dose, because the chamber measures the total absorbed dose and also Q4 characterizes radiation quality of mixed radiation field. The original data were given with accuracy of about $20 \%$, but the re-calculation introduces additional uncertainty associated with uncertainty of Qref, so the total uncertainty is of about 25\%. Disagreement at such level could be accepted for radiation protection (sometimes the factor of 2 in data have to be taken).

Comparison of the calculated and experimental values of the dose absorbed in the gas cavity relatively to $\mathrm{D}^{*}(10)$ is shown in Fig. 4. Uncertain-

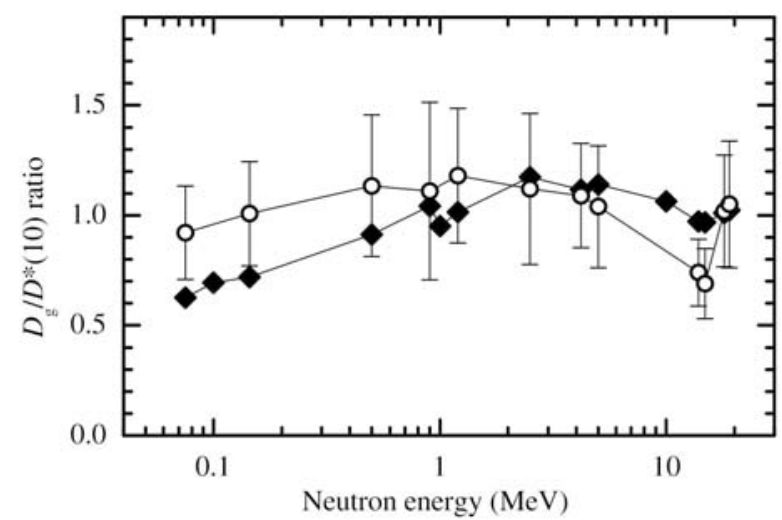

Fig. 4. Calculated (diamonds) and measured (circles) dose absorbed in gas $(\mathrm{Dg})$ to $\mathrm{D}^{*}(10)$ ratio as a function of neutron energy for monoenergetic neutrons. 
ties have been estimated and indicated for each point.

\section{Discussion}

Composition of the gas filling the recombination chamber should be optimized taking into account three desirable features of the chamber response:

- The energy dependence of the chamber response to $\mathrm{H}^{*}(10)$ of neutrons should be as flat as possible in whole energy range;

- Recombination index of radiation quality should have values well approximating $\mathrm{Q}^{*}(10)$;

- The neutron sensitivity of the detector should be similar to the photon sensitivity.

The choice of tissue equivalent (TE) gas results in too low relative sensitivity to neutrons (about 10\% lower than to photons). Recombination of ions in TE gas is relatively high, so accurate determination of saturation current can be difficult. For these reasons, other gases with lower density have been considered and the mixture of $\mathrm{CH}_{4}$ with $\mathrm{N}_{2}$ (5\% by weight) has been chosen for commercially manufactured chambers. Neutron sensitivity (ratio of the saturation current to $D^{*}(10)$ ) of the chamber filled with such mixture is about $15 \%$ higher than sensitivity to photons, for neutron energy range up to about $5 \mathrm{MeV}$. This is associated with high excess of hydrogen contents in the gas comparing to soft tissue. The mixture with 5\% of nitrogen contains $23.75 \%$ of hydrogen, whereas TE gas contains only $11.1 \%$. Increasing addition of nitrogen in gas mixtures, investigated in this work, resulted in gradual decrease in the dose absorbed in the gas. The mixture with $50 \%$ of nitrogen contains $12.5 \%$ of hydrogen - still more than TE gas. Clear dependence of the dose absorbed in the gas on the content of hydrogen indicates that, for the irradiation geometry assumed in this work, the majority of neutron interactions in large high pressure ionization chamber occurs in the gas cavity.

There is a considerable increase in the dose to the gas at low neutron energies. In radiation fields with broad energy spectra, this effect may compensate for the lower response for intermediate and low energy neutrons.

Figures 2 and 3 show that dose absorbed in the gas is generally lower than $\mathrm{D}^{*}(10)$, most likely because of larger effective thickness of the chamber wall, comparing with the $10 \mathrm{~mm}$ depth of the reference point in ICRU sphere. In practice, this underestimation is well compensated by proper calibration of the chamber.

The calculated energy dependence of the chamber response to $D^{*}(10)$ is in acceptable agreement with the experimental data for monoenergetic neutrons, despite different irradiation geometries.

\section{Conclusions}

First simulation of the REM-2 chamber with MCNPX code made it possible to compare the energy dependence of the chamber response to $D^{*}(10)$ for different filling gases. The results showed clear dependence of the dose absorbed to the gas on hydrogen content. The shape of the energy dependence is similar for all the gases investigated in the work.

In future studies, changes in the chamber construction will be proposed. At this stage of development, it could be stated that, in the case of constructing the chamber for low energy neutron detection, the amount of nitrogen in gaseous mixture filling the chamber has to be raised to several tens of percent, or small amount of boron addition to filling gas could be taken into consideration.

Acknowledgment. This work is partly supported by the funds of the National Science Centre grant "Recombination dose meter of new generation for exposure assessment on workplaces in radiation fields of reactors and accelerators" [1350/B/P01/2010/39].

\section{ORCID}

Katarzyna Tymińska (D) http://orcid.org/0000-0002-5702-2708 Michat A. Gryziński (D http://orcid.org/0000-0002-4860-8176 Maciej Maciak (1) http://orcid.org/0000-0003-3842-6720

\section{References}

1. Zielczyński, M., Golnik, N., \& Rusinowski, Z. (1996). A computer controlled ambient dose equivalent meter based on a recombination chamber. Nucl. Instrum. Methods Phys. Res. Sect. A-Accel. Spectrom. Dect. Assoc. Equ., 370(2/3), 563-567. https://doi. org/10.1016/0168-9002(95)01013-0.

2. Zielczyński, M., \& Golnik, N. (1994). Recombination index of radiation quality - measuring and applications. Radiat. Prot. Dosim., 52, 419-422. https://doi. org/10.1093/oxfordjournals.rpd.a082226.

3. Golnik, N., Gryziński, M. A., Kowalska, M., Meronka, K., \& Tulik, P. (2014). Characterization of radiation field for irradiation of biological samples at nuclear reactor - comparison of twin detector and recombination methods. Radiat. Prot. Dosim., 161(1/4), 196-200. DOI: 10.1093/rpd/nct341.

4. Golnik, N. (1996). Recombination methods in the dosimetry of mixed radiation. Swierk, Poland: Institute of Atomic Energy. (Report IEA-20/A).

5. Golnik, N., Silari, M., \& Otto, T. (1999). On the use of a recombination chamber for radiation measurements in CERN-EU high-energy reference radiation fields. Radiat. Prot. Dosim., 86(3), 175-179. https:// doi.org/10.1093/oxfordjournals.rpd.a032940.

6. Silari, M., Agosteo, S., Beck, P., Bedogni, R., Cale, E., Caresana, M., Domingo, C., Donadille, L., Dubourg, N., Esposito, A., Fehrenbacher, G., Fernandez, F., Ferrarini, M., Fiechtner, A., Fuchs, A., Garcia, M. J., Golnik, N., Gutermuth, F., Khurana, S., Klages, Th., Latocha, M., Mares, V., Mayer, S., Radon, T., Reithmeier, H., Rollet, S., Roos, H., Ruhm, W., Sandri, S., Schardt, D., Simmer, G., Spurny, F., Trompier, F., Villa-Grassa, C., Weitzenegger, E., Wiegel, B., Wielunski, M., Wissmann, F., Zechner, A., \& Zielczyński, M. (2009). Intercom- 
parison of radiation protection devices in a high-energy stray neutron field. Part III: Instrument response. Radiat. Meas., 44(7/8), 673-691. DOI: 10.1016/j. radmeas.2009.05.005.

7. Golnik, N., Brede, H. J., \& Guldbakke, S. (1997). $\mathrm{H}^{*}(10)$ response of the REM-2 recombination chamber in monoenergetic neutron fields. Radiat. Prot. Dosim., 74(3), 139-144. https://doi.org/10.1093/ oxfordjournals.rpd.a032189.

8. International Commission on Radiation Units and Measurements. (1983). Microdosimetry. ICRU Publications. Bethesda. (ICRU Report 36).
9. International Commission on Radiation Units and Measurements. (1993). Quantities and units in radiation protection dosimetry. ICRU Publications. Bethesda. (ICRU Report 51).

10. Leuthold, G., Mares, V., \& Schraube, H. (1992). Calculation of the neutron ambient dose equivalent on the basis of the ICRP revised quality factors. Radiat. Prot. Dosim., 40(2), 77-84. 